\title{
To Developed Tool, an Intelligent Agent for AutomaticKnowledge Acquisition In Rule-based Expert System
}

\author{
Mohammed Abbas Kadhim \\ Dept. of Computer Science, Hamdard University \\ Hamdard Nagar, New Delhi-110062, India
}

\author{
M. Afshar Alam \\ Dept. of Computer Science, Hamdard University \\ Hamdard Nagar ,New Delhi-110062, India
}

\begin{abstract}
The complex stage in building expert system is knowledge acquisition from domain experts and translated it to representation approach in knowledge base of expert system. In this paper we present an automatic way to extract knowledge from domain experts directly and converted it to facts and rules in knowledge base for rule-based expert system using intelligent agent. That means, the construct of proposed system consists of three steps, in first step we are construct a Diagnosis Domain Tool for Expert System (DDTES) (a piece of software which contains the user interface, inference engine and a format for declarative knowledge in knowledge base). Secondly we are construct Knowledge Acquisition Agent (KAA) able to interview with domain experts to extract problem solving knowledge in specific diagnosis domain and converted it to production rules in knowledge base. Finally we captured complete rule-based expert system from combination the results of two previous steps which can works and produce advices in that domain. The proposed (DDTES) and (KAA) are implemented and executed by using Visual Prolog programming language Ver.7.1.
\end{abstract}

\section{General Terms}

Artificial intelligent, intelligent agent, expert system.

\section{Keywords}

Knowledge acquisition agent, automatic knowledge acquisition, expert system tools, rule-based expert system.

\section{INTRODUCTION}

Computer system has become a part of our everyday lives, Artificial Intelligence (AI) is sometimes refer to machine intelligence which is concerned with devising computer programs to make computer more intelligent, expert system is one of the most common application of A.I., it is a computer program that simulates the judgment and behavior of a human or an organization that has expert knowledge and experience in a particular field. Typically, such a system contains knowledge base containing accumulated experience and a set of rules for applying the knowledge base to each particular situation that is described to the program [1], the other components for expert system as well as the knowledge base are user interface and inference engine, the process of acquiring knowledge from experts and building knowledge base is called knowledge engineering [2]. In this research, we focus on construct a diagnosis domain tool for building expert system and automatic constructed of knowledge base by using intelligent agent approach.

The term expert system tools describes the software system that is used for constructing an expert system [3], most expert systems are developed using specialized software tools called shells. These shells provided with an inference mechanism such as backward chaining, forward chaining or both, and require knowledge to be entered according to a specified format representation [4]. Shells are probably the most common development environment tool. They can be categorized as general or domain specific, domain-specific tools are designed to be used only in the development of an expert system for a specific domain application area for example, there are shells for diagnostic systems, configuration, and financial applications [2].

Agents are special types of software applications, different from non-agent software applications in their ability to run autonomously, in last decade agent become a very popular paradigm in computing. The reasons for this popularity is their flexibility, modularity and general applicability to a wide range of problems [5]. The concept of an agent play a main role in both artificial intelligence approach and mainstream of computer science, recently the use of intelligent agents has been applied in different application area such as in userfriendly interface system, vision system, knowledge discovery and data mining, and information retrieval system, the agent has been introduced, in the form of an agent that can act in the place of a human being[6,7].

Agents can be classified into two major categories: resident and mobile. Resident agents stay in the computer or system and perform their tasks there. Mobile agents move to other systems, performing tasks there. A mobile agent can transport itself across different system architectures [8].

In this study we produce a rule-based expert system can works and produce advice in diagnosis domain depend on, firstly the domain specific tool for building expert system which responsible for constructing user interface and inference engine which used backward chaining strategy for reasoning process for rule-based expert system and, secondly is constructing an intelligent agent which is able to interact with domain experts to extract expert's problem solving knowledge as production rules in diagnosis domain and put it in knowledge base for that system. 


\section{RELATED WORKS}

Recently, a number of studies have been proposed and implemented using expert system tools (shells) and intelligent agents or multi-intelligent agents in different domains, we will review a set of this studies which concern with knowledge acquisition approaches and construct expert systems.

In [9], The authors describes JavaDON, an open-source expert systems shell based on the OBOA framework for developing intelligent systems. The central idea of the JavaDON project was to make an easy-to-use and easy-to-extend tool for building practical expert systems. Since JavaDON is rooted in a sound theoretical framework, it is well-suited for building even complex expert system applications, both stand alone and Web-based ones. In [10], they present a dynamic, uncertainty-based user model knowledge representation for use in an intelligent interface agent called GESIA. They critically discuss the construction of user models, relating their construction with user intent and the relevancy of observable properties in the user's "world." Their agent's usefulness is demonstrated in the domain of a generic expert system shell called PESKI.

In [11], The work described learning and communication between cognitive artificial agents. Focus is on dialogue as the only way for agents to acquire knowledge, as it often happens in natural situations. Since this restriction has scarcely been studied as such in artificial intelligence (AI), until now, this research aims at providing a dialogue model devoted to knowledge acquisition. In [12], multi-agent technology is utilized in implementation of Enterprise Resource Planning (ERP) system to improve the capability of knowledge acquisition and flexibility of the system. In [13] describes extending the automated discovery mechanism of the Knowledge Encapsulation Framework (KEF) through the use of agent technology. In [14], they proposed an intelligent agent model for smart home environments. In ubiquitous computing environments like smart home, agents have to learn user's preferences in order to assist them. These preferences are represented by user profiles

In [15], produce a formal model of agent-based knowledge management in intelligent tutoring systems. It consists of three agents, a knowledge acquisition agent, a knowledge distribution agent and a knowledge maintaining agent. The knowledge acquisition agent is responsible for the construction of user model and domain knowledge base. In [16], the authors proposed architecture of multi intelligent agents for crop suitability based on irrigation water quality identifies the quality of water for the purpose of irrigation and also guide user about suitable crops related to that water quality.

\section{PROPOSED SYSTEM}

A rule-based expert system is an expert system based on a set of rules that a human expert follow in diagnosing a problem [4]. In our study we proposed a rule-based expert system produce advices in specific diagnosis domain. The construction of proposed system consists of three phases as follow:

\subsection{Diagnosis Domain Tool for Expert System (DDTES)}

Recently, there are different expert system development tools. They differ in the level of flexibility they provide in building expert system and in the range of knowledge representation methods, reasoning chaining, and other intelligent techniques they support [9]. An expert system tool, or shell, is a software development environment containing the basic components of expert systems. Associated with a shell is a prescribed method for building applications by configuring and instantiating these components [3]

The proposed diagnosis domain tool system is an expert system with emptied knowledge base (or without knowledge base) as in figure (1) can works in diagnosis domain, that means this system consist of only from user interface and inference engine the developers can concentrate only on entering the knowledge base (discuss in next section).

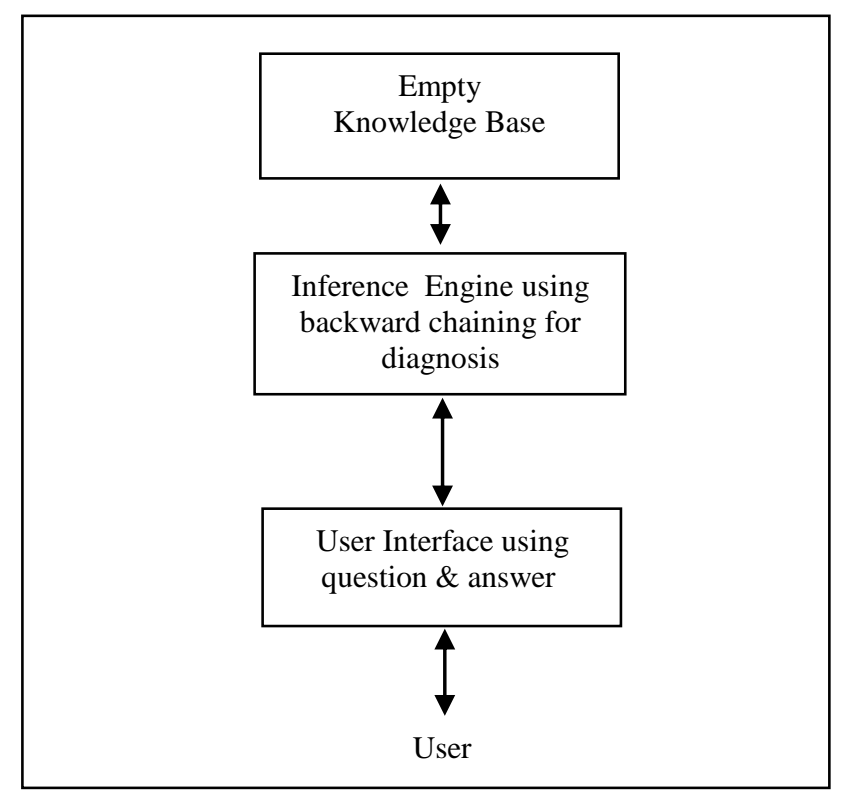

Figure 1 :Components of DDTES

The user can interact with expert system through User Interface (U.I), the U.I must be friendly of user and hide the other complex components of expert system, the questions and answers method used in building of user interface for the system.

The Inference Engine (I.E) of the system can recognize the stored knowledge based on the method used for knowledge representation (production rules), the I.E contain the strategies of reasoning process, its carries out the reasoning process by links the contains of the knowledge base with the symptoms which input by users through user interface to capture appropriate decision situation. the backward chaining strategy is used in inference engine of proposed system.

\subsection{Knowledge Acquisition Agent (KAA)}

Knowledge base is one of the main component of expert system with the main functions of storing and managing the knowledge in expert system. The performance of an expert system mainly depends on the quantity and quality of knowledge that the system contains, therefore the representation and access to knowledge are play main role in the development and use of an expert system [17]. Knowledge acquisition is the process of acquiring knowledge either directly from a human experts or from some other resources, and its formalized structure, that will allow some particular task to be performed by a computer system [15].

Software agents are computer programs different from nonagent programs in their ability to run autonomously sensing and acting on changing environment condition because they run autonomously [8]. The intelligent agent system which is used for knowledge acquisition become more efficient when we are restricted to a particular problem solving domain. In proposed agent we are restricted to a diagnosis domain using production rules to represent knowledge in knowledge base 
the diagnosis is one of general expert system problem categories which means determining the cause of malfunction in complex situation based on observable symptoms, that mean the output of proposed agent is a knowledge base in specific diagnosis domain. The following paragraphs show the main components of (KAA) :

\subsubsection{Expert interface}

The proposed agent can be interacted with domain experts by interface, the interface of proposed agent must be friendly of expert and more comfortable for the human and must be hide the complexity of other components of agent. The interface built by using menu driven technique and questions and answers in natural language to become closest to expert. Figure(2) illustrate main components of (KAA). The interface also permits for experts to be update of knowledge in knowledge base. That means the ability of interact combination between adding knowledge and previous contains of knowledge base. The output of interface is knowledge description from the input conceptual description.

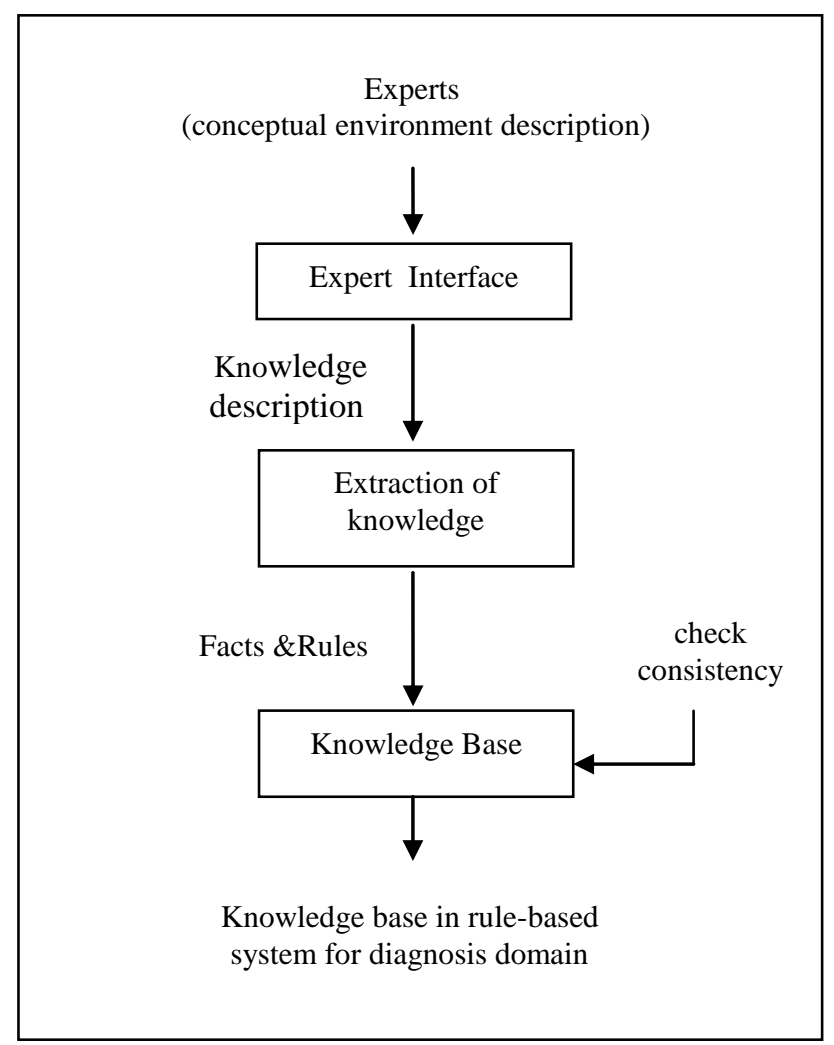

Figure 2 : Main components of KAA

\subsubsection{Extract of knowledge}

After knowledge description, the second phase has been started which involves extracting facts and rules from knowledge description. The fact is a relationship between two objects (or more) without condition, while the rule consist of two elements, the first one (action or head of rule) is the relationship between two objects (or more), the other one is the conditions (body of the rule) which is the set of relations that must be satisfied to carry out of head of that rule (action). In diagnosis domain the head of rule represent the complex situation and the body of rule represent the observed symptoms. After extraction, knowledge (facts and rules) will be written in knowledge base as clauses in Prolog programming language.

\subsubsection{Knowledge base}

Before knowledge (facts and rules) have been written in knowledge base the (KAA) must be check that facts and rules consistency with other contains of knowledge base (consistency means there is no confliction between the new knowledge and exist knowledge in knowledge base). In general the following algorithm represent the main steps of proposed agent:

\section{Algorithm}

1-Input the main situation which want to diagnosis as string variable name $D$.

2-IF the knowledge base is empty THEN continue ELSE go to step 5 .

3-Input new symptoms as $\left(A_{1}, A_{2} \ldots A_{r}\right), r$ is the no. of new symptoms.

4-Construct new rule as (D:- $\left.A_{1}, A_{2}, \ldots A_{r}\right)$ go to step 10.

5-Display all the symptoms $\left(\mathrm{S}_{1}, \mathrm{~S}_{2}, \ldots \mathrm{S}_{\mathrm{n}}\right)$ in knowledge base in order to select appropriate symptoms $\left(\mathrm{C}_{1}, \mathrm{C}_{2}, \ldots \mathrm{C}_{\mathrm{m}}\right)$ for input situation $\mathrm{D}$ by domain expert, where $\mathrm{n}$ and $\mathrm{m}$ are no. of all symptoms and no. of selected symptoms respectively.

6-IF you have new symptoms THEN continue ELSE go to step 9.

7-Input these new symptoms $\left(A_{1}, A_{2}, \ldots A_{x}\right)$, where $x$ is the no. of new symptoms.

8-Construct a new rule as $\left(\mathrm{D}:-\mathrm{C}_{1}, \mathrm{C}_{2}, \ldots \mathrm{C}_{\mathrm{m}}, \mathrm{A}_{1}, \mathrm{~A}_{2}, \ldots \mathrm{A}_{\mathrm{x}}\right)$ and go to 10

9- Construct a new rule as (D:- $\left.\mathrm{C}_{1}, \mathrm{C}_{2}, \ldots \mathrm{C}_{\mathrm{m}}\right)$

10-IF find confliction THEN go to step 12 ELSE continue

11-Write this rule in knowledge base as a clause in Prolog programming language 12-End

\subsection{Complete Rule-based Expert System}

When the construct of knowledge base is the bottleneck of construct of rule-based expert system, therefore the proposed agent focuses on construct of knowledge base. In other words, the main task of proposed intelligent agent is to build the knowledge base in rule-based expert system. The other components of rule-based expert system such as user interface and inference engine are constructed by the proposed expert system tool as in figure (3) which represent complete rulebased expert system with DDTES and KAA, now we captured complete rule-based expert which produce advices in specific diagnosis domain (depend on the contains of knowledge base) such as medical diagnosis domain (back pain diseases, eye diseases...etc) or non-medical diagnosis domain (organic chemical, inorganic chemical diagnosis... etc). 


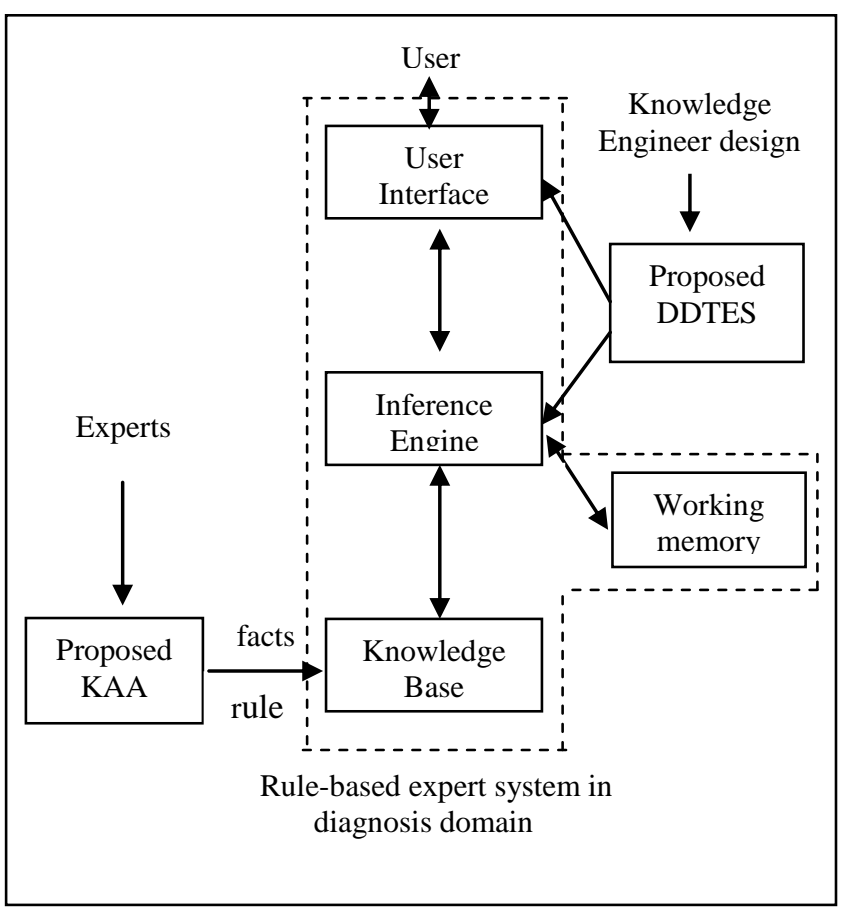

Figure 3 : Complete rule-based expert system with DDTES and KAA

as questions, the user answers for that questions by YES or $\mathrm{NO}$ if the certain symptom appear or not. In the end, depend on the user's answers the name of situation posted up on the screen. The system work like this: the system display first symptom (the symptom that most frequency in all situations in working memory) if it has positive answer then will keep with all situations has this symptom and eliminate all situations which don't has this symptom from working memory and vise versa, then display the second symptom (the symptom that most frequency in all situations in working memory) if it has positive or negative answer then will keep or eliminate the situations that has or don't has this symptom, repeat that procedure until to arrive the specific situation. The following algorithm illustrate that works:

\section{Algorithm}

1-Load all situations and its symptoms from knowledge base to working memory.

2-Display the most frequently symptom in all situation in working memory as a question

3-IF answer is Yes THEN continue ELSE go to step 6

4-Keep all situations that has this symptom and remove all situations that don't has this symptom from working memory, go to step 7

6-Remove all situations that has this symptom from working memory

7-IF working memory contain one situation THEN continue ELSE go to step 2

8-Display this situation as advice (decision) to user 9-End

\section{IMPLEMENTATION AND CASE STUDY}

The proposed system is developed using Visual Prolog 7.1 programming language. The tests of the system were performed using medical diagnosis for back pain diseases (we can use any diagnosis domain), the implementation of this case study can be do it through :

\subsection{Implementation of KAA}

To evaluate the working of the KAA system, we will discuss the interaction between expert and proposed agent to show the steps of system carry out to produce knowledge base for back pain diagnosis. The KAA produce many of questions to expert such as the situation name (disease name) and a set of symptoms which satisfy that situation, figure (4) and (5) illustrated main steps implementation of KAA.

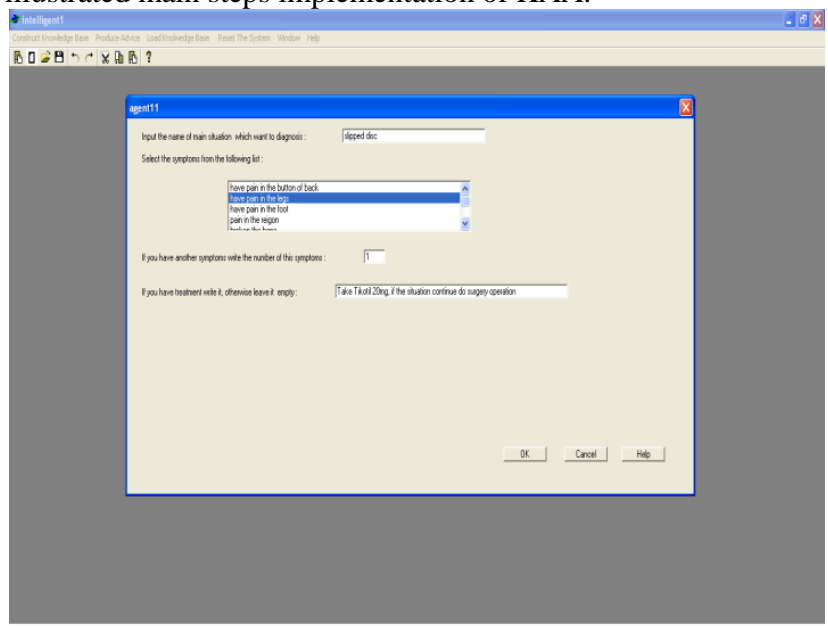

Figure 4 :Input disease name and its overlap symptoms

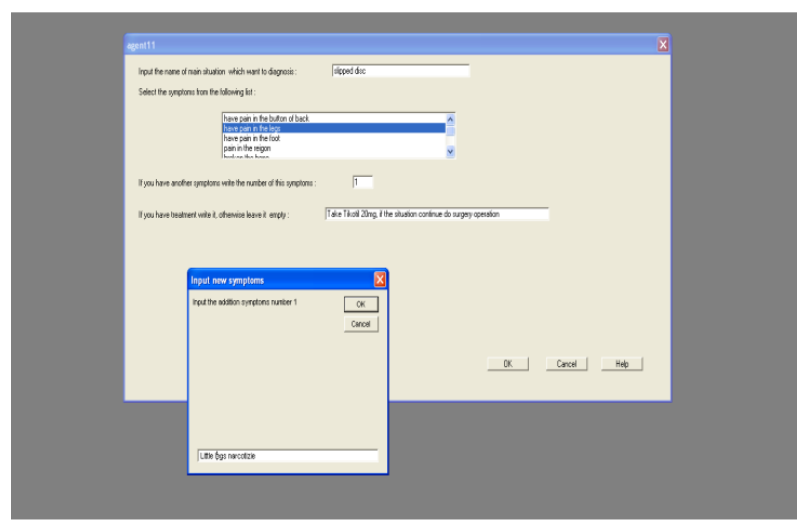

Figure 5 :Input disease name and its new symptoms

After complete this interaction between expert and KAA, the KAA translate the acquired data to facts and rules clauses in Prolog language and write it in knowledge base, figure (6) illustrate sample of facts and rules extracted from above case and written in knowledge base.

name_disease("slipped disc"):-cond3,cond4,cond10,cond12. condition (cond3, "have pain in the button of back bone"). condition (cond4," have pain in your legs"). condition (cond10,"foot senseless"). condition (cond12,"little legs narcotize "). diagnosis ("slipped disc",[cond3, cond4, cond10,cond12]) treatment("slipped disc", "Take drugs like Tilcotil20mg, if the situation continue do surgery operation").

Figure 6 : Sample facts and rules in knowledge base

\subsection{Implementation of Rule-based Expert System}

After complete the construction of knowledge base by KAA, the rule-based expert system can work and produce advices to 
users (patients) are infected with back pain diseases, the figure (7) and (8) illustrated the interaction between the patient and expert system to arrive appropriate advices (decisions).

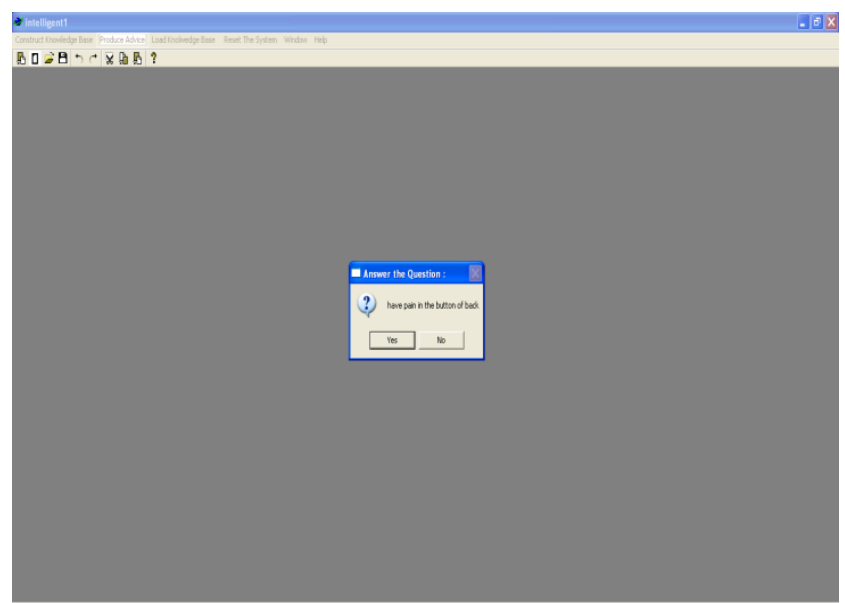

Figure 7 :Interaction between patient and expert system

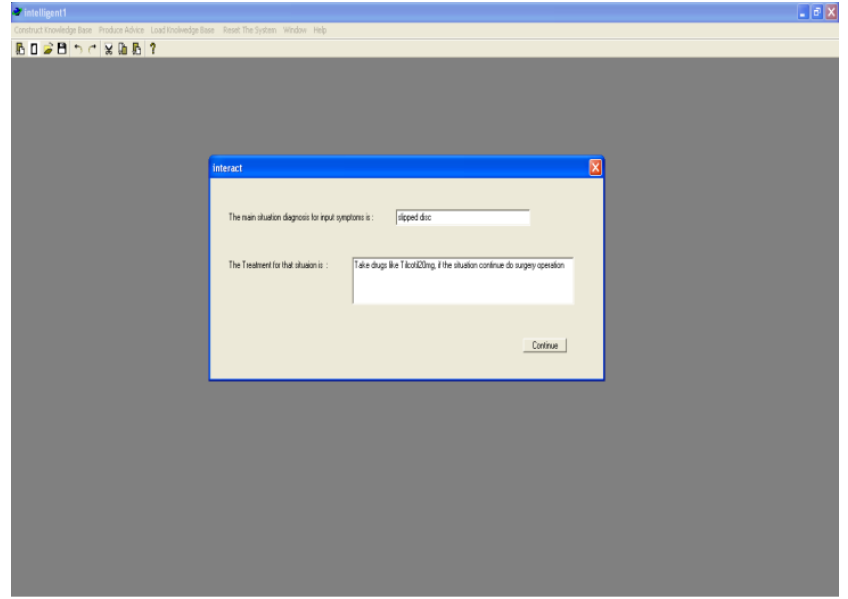

Figure 8 :Produce advice by expert system

\section{CONCLUSION}

This paper discuss the architecture of DDTES and KAA to obtain rule-based expert system in diagnosis domain. By applying intelligent agent approach for extracting knowledge from experts we increase power and flexibility of the existing framework, the proposed agent used strategies to learn new knowledge and discover conflicts between new knowledge and stored knowledge furthermore, it can quick up construct of rule-based expert system and reduces the amount of time that an expert may take when try to explain his experience to knowledge engineer, that means instead of using traditional methods to extract knowledge we can use intelligent agent system for that job, also we discussed a general model for constricting tool for building expert system and resident intelligent agent in diagnosis domain. Finally, we used one application of artificial intelligent (intelligent agent) to construct another application (expert system).

\section{REFERENCES}

[1] Luger, F. and William Stubbefield , 1998, Artificial Intelligence, Addison Wesley Longman, $3^{\text {rd }}$ edition.

[2] http://wps.prenhall.com/wps/media/objects/3778/3869053/ Turban Online Chapter W18. pdf, Knowledge Acquisition, Representation, and Reasoning.
[3] Eldrandaly,Khalid, October 2007, An Intelligent MCDM Approach for Selection the Suitable Expert System Building Tool, The International Arab Journal of Information Technology, Vol. 4, No. 4.

[4] Abraham,Ajith, 2005, Rule-based Expert Systems, John Wiley \& Sons.

[5] Yasemin,Ayse, , May 1999, Intelligent Agents: Data Mining Perspective, http://citeseerx.ist.psu. Edu/viewdoc /download?doi=10.1.1.23... pdf.

[6] Kim,D., Chang Suk Kim, and Kee Wook Rim, June 2003, Modeling and Design of Intelligent Agent System, International Journal of Control, Automation, and Systems Vol. 1, No. 2, pp 257-261.

[7] Wooldridge, M. and Nicholas R., October 1994, Intelligent Agent : Theory and Practice, Knowledge Engineering review.

[8]http://jpkc.whut.edu.cn/dzswgl/\%E7\%94\%B5\%E5\%AD\% 90\%E8\%AF\%BE\%E4\%BB\%B6/Cases \%20 (16).pdf , Software (Intelligent) Agents.

[9] Tomic, 'Bojan, Jelena Jovanovic' and Vladan Devedz ic, 2006, JavaDON: an open-source expert system shell, Expert Systems with Applications, Elsevier, pp 595606.

[10] Brown,S. M., Robert A. Harrington, Eugene Santos Jr. and Sheila B. Banks, 2008, User Models, Intelligent Interface Agents and Expert Systems, http://citeseerx.ist.psu.edu/viewdoc/download? doi=10.1.1.31.945

[11] Yousfi-Monod,M. and Violaine Prince, Knowledge Acquisition Modeling Through Dialogue Between Cognitive Agents, http://citeseerx.ist.psu.edu/viewdoc/summary?doi=10.1.1 .103 .7827

[12] Chen, Liang and Jianmin Gao, August 2004, Knowledge Acquisition System Based-on Multi-agent Technology in ERP Implementation Assistant, International Conference on intelligent Mechatronlcs and Automation Chengdu, China, pp 152-156.

[13] Haack, J., Andrew Cowell, Eric Marshall, Keith Fligg, Michelle Gregory and Liam McGrath, 2009, AgentBased Knowledge Discovery for Modeling \& Simulation, IEEE/WIC/ACM International Joint Conferences on Web Intelligence and Intelligent Agent Technologies, pp 543-546.

[14] Kushwaha,Neeraj, Minkoo Kim, Dong Yoon Kim and We-Duke Cho, 2004, An Intelligent Agent For Ubiquitous Computing Environments: Smart Home UTAGENT, The Second IEEE Workshop on Software Technologies for Future Embedded and Ubiquitous Systems (WSTFEUS'04).

[15] Zhiping,L., XU Tianwei and SUN Yu, , 2010 IEEE, Agent-based Knowledge Management in Intelligent Tutoring Systems, The 5th International Conference on Computer Science \& Education Hefei, China. August 24-27, pp 578-582.

[16] Sharma, Priyanka, Jan. 2010, Architecture of MultiIntelligent Agents for Crop Suitability Based on Irrigation Water Quality, International Journal of Advanced Engineering \& Applications, pp 163-169.

[17] Rui,W. and Long Duo, 2011, The Study on Construction of Knowledge Base of Grinding Expert System Based on Data Mining, International Conference on Mechatronic Science, Electric Engineering and Computer, August 1922, Jilin, China, pp 845-848. 